\title{
How antibiotic resistance is shaped by host- microbiome interactions in influenza
}

Lingdi Zhang

Christian V. Forst

Aubree Gordon

Gabrielle Gussin

Adam B. Geber

Porfirio J. Fernandez

Tao Ding

Lauren Lashua

Minghui Wang

Angel Balmaseda

Richard Bonneau

Bin Zhang

Elodie Ghedin

\section{Video Byte}

Keywords: antibiotic resistance, upper respiratory tract infection, microbiome, influenza, metatranscriptome, immunology, virus, Microbiome

Posted Date: May 4th, 2020

DOI: https://doi.org/10.21203/rs.3.rs-27126/v1

License: (9) This work is licensed under a Creative Commons Attribution 4.0 International License. Read Full License 


\section{Abstract}

Influenza infection can be tough to fight on its own, but secondary bacterial infections can make influenza even more serious, especially among elderly and immunocompromised individuals. These bacteria have the potential to become antibiotic resistant, making treatment even more complicated. Unfortunately, the expression of antibiotic resistance genes (ARGs) by microbes in the respiratory tract is not well understood. A recent study sought to characterize ARG expression in microbes during influenza infection. Researchers evaluated nasal swab samples and blood samples from 37 influenza-infected patients. Sequencing revealed that ARG expression was particularly common in S. aureus and S. pneumoniae species in the airway, and host transcriptome profiling identified several host key regulators involved in the influenza response, suggesting that the respiratory tract may be an important reservoir of ARGs in humans. Although further study is needed, the study highlights critical correlations between the host response to influenza and the secondary bacteria present in the airway, improving our understanding of a critical aspect of the interplay between host and microbe in influenza infection. 